\title{
A Schur-Like Basis of NSym Defined by a Pieri Rule
}

\author{
John Campbell Karen Feldman Jennifer Light \\ Pavel Shuldiner Yan Xu \\ York University \\ Ontario, Canada \\ \{maxwell8, chagit, lightj, pavelshu, simonxiu\}@yorku.ca
}

Submitted: Nov 2, 2013; Accepted: Sep 2, 2014; Published: Sep 18, 2014

Mathematics Subject Classifications: 05E05, 05A05, 05A17

\begin{abstract}
Recent research on the algebra of non-commutative symmetric functions and the dual algebra of quasi-symmetric functions has explored some natural analogues of the Schur basis of the algebra of symmetric functions. We introduce a new basis of the algebra of non-commutative symmetric functions using a right Pieri rule. The commutative image of an element of this basis indexed by a partition equals the element of the Schur basis indexed by the same partition and the commutative image is 0 otherwise. We establish a rule for right-multiplying an arbitrary element of this basis by an arbitrary element of the ribbon basis, and a Murnaghan-Nakayama-like rule for this new basis. Elements of this new basis indexed by compositions of the form $\left(1^{n}, m, 1^{r}\right)$ are evaluated in terms of the complete homogeneous basis and the elementary basis.
\end{abstract}

\section{Introduction}

The Hopf algebra Sym of symmetric functions is a subalgebra of QSym, the Hopf algebra of quasi-symmetric functions. By duality there exists a projection map $\chi:$ NSym $\rightarrow$ Sym, where NSym denotes the Hopf algebra of non-commutative symmetric functions, which is dual to QSym. The bases of NSym and QSym are indexed by the set of integer compositions $\mathcal{C}$ and the bases of Sym are indexed by the set of integer partitions $\mathcal{P}$. A natural question to ask is whether there exists a basis of NSym which projects in a natural way onto the Schur basis of Sym.

From the time of the introduction of QSym [Gessel, MR] and NSym [NCSF], it was generally accepted that the closest analogues of the Schur basis were the "fundamental" basis 
$\left\{F_{\alpha}\right\}_{\alpha \in \mathcal{C}}$ of QSym and (its dual) the "ribbon" basis $\left\{R_{\alpha}\right\}_{\alpha \in \mathcal{C}}$ of NSym. The fundamental basis has the property that

$$
s_{\lambda}=\sum_{\operatorname{shape}(P(\sigma))=\lambda} F_{D(\sigma)}
$$

where the notation indicates that the sum is over all permutations such that the shape of the $P$-tableaux (in the RSK correspondence) is equal to $\lambda$ and $D(\sigma)$ is the descent composition of the permutation $\sigma$. The ribbon basis has the property that

$$
\chi\left(R_{\alpha}\right)=s_{\nu / \bar{\nu}}
$$

where $\nu / \bar{\nu}$ is a skew partition representing a right alignment of the rows of the composition $\alpha$.

The position of the fundamental and ribbon bases as "the" Schur analogues of QSym and NSym was called into question in the exploration of the quasi-symmetric function expansion of Macdonald polynomials [HHL]. The quasi-symmetric expansion seemed to lead to the definition of a quasi-symmetric basis, $\left\{\mathcal{S}_{\alpha}\right\}_{\alpha \in \mathcal{C}}$, that was a close analogue of the Schur basis and so it was named "quasi-Schur" [HLMvW11a]. It was noted that this basis was very closely related to the Demazure atoms [LS] of Lascoux and Schützenberger and the quasi-Schur basis of QSym was a natural analogue of the Schur symmetric functions because:

$$
s_{\lambda}=\sum_{\substack{\alpha \in \mathcal{C} \\ \operatorname{sort}(\alpha)=\lambda}} \mathcal{S}_{\alpha} .
$$

The dual quasi-Schur basis $\left\{\mathcal{S}_{\alpha}^{*}\right\}_{\alpha \in \mathcal{e}}$ of $[\mathrm{BLvW}]$ has the property that

$$
\chi\left(\mathcal{S}_{\alpha}^{*}\right)=s_{\text {sort }(\alpha)} .
$$

More recently, Berg et. al. [BBSSZ] introduced a second analogue of the Schur functions, which they dubbed the "immaculate" basis of NSym, $\left\{\mathfrak{S}_{\alpha}\right\}_{\alpha \in \mathfrak{e}}$, as well as the dualimmaculate basis of QSym $\left\{\mathfrak{S}_{\alpha}^{*}\right\}_{\alpha \in \mathcal{e}}$. The immaculate basis of NSym satisfies the following analogue of the Jacobi-Trudi rule for the Schur basis:

$$
\mathfrak{S}_{\alpha}=\sum_{\sigma \in S_{\ell(\alpha)}} \operatorname{sign}(\sigma) H_{\alpha_{1}+\sigma_{1}-1, \alpha_{2}+\sigma_{2}-2, \ldots, \alpha_{\ell(\alpha)}+\sigma_{\ell(\alpha)}-\ell(\alpha)}
$$

Therefore,

$$
\chi\left(\mathfrak{S}_{\alpha}\right)=\operatorname{det}\left[h_{\alpha_{i}+j-i}\right]_{\ell(\alpha) \times \ell(\alpha)}
$$

and in particular for a partition $\lambda, \chi\left(\mathfrak{S}_{\lambda}\right)=s_{\lambda}$. The dual immaculate basis has the property that

$$
s_{\lambda}=\sum_{\sigma} \operatorname{sign}(\sigma) \mathfrak{S}_{\lambda+\mathrm{id}-\sigma}^{*}
$$

where the addition is coordinate-wise as vector entries, and id represents the identity permutation and the sum is over permutations $\sigma$ of $\{1,2, \ldots, \ell(\lambda)\}$ such that $\lambda+\mathrm{id}-\sigma$ is a composition. 
Computer explorations of NSym and QSym using the software Sage [sage] lead us to consider other related possible bases of NSym and their dual bases of QSym. We are ideally searching for a basis that simplifies proofs of Schur-positivity properties of quasisymmetric function expansions. A combinatorial algorithm of this type may or may not be possible; however, there are reasons to believe that each of these bases of NSym and QSym closely reflect different combinatorial aspects of compositions and composition tableaux and their relationship to partitions and column strict tableaux.

In this paper, we introduce a basis $\left\{\boldsymbol{\Psi}_{\alpha}\right\}_{\alpha \in \mathcal{C}}$ of NSym called the shin basis as another analogue of the Schur basis, together with a dual basis $\left\{\boldsymbol{\Psi}_{\alpha}^{*}\right\}_{\alpha \in \mathcal{C}}$ of QSym. They are defined so that they have the simplest combinatorial rule we could identify, with the property that

$$
\chi\left(\boldsymbol{\Psi}_{\alpha}\right)=\left\{\begin{array}{ll}
s_{\lambda} & \text { if } \alpha=\lambda \in \mathcal{P} \\
0 & \text { otherwise }
\end{array} .\right.
$$

Dually, we have that for a partition $\lambda$,

$$
s_{\lambda}=\dddot{\Psi}_{\lambda}^{*} .
$$

This implies that $\boldsymbol{\Psi}_{\alpha}^{*}$ is a symmetric function if and only if $\alpha$ is a partition. We have chosen to develop this basis of NSym because many of the algebraic properties that we have discovered are more clearly stated on the basis $\boldsymbol{\Psi}_{\alpha}$ than the basis $\boldsymbol{\Psi}_{\alpha}^{*}$.

After a few preliminary definitions, we introduce a definition of the shin basis in Section 3. In the next section, we prove a combinatorial formula for the product of an element of the shin basis and an element of the ribbon basis, and in Section 5 we prove a combinatorial formula for the product of an element of the shin basis and an element $\Psi_{n}$, which is an analogue of the power sum generator of degree $n$ for NSym. In Section 6 , we give a formula for the shin function evaluated at compositions of the form $\left(1^{n}, m, 1^{r}\right)$. We give an explicit definition of the graded dual of the shin basis, a basis of QSym. We show the close relationship between the shin function and the Schur basis of Sym.

\section{Acknowledgments}

This work has been completed as part of an undergraduate research project at York University. The authors would like to thank Mike Zabrocki for supervising this summer research project and providing useful comments reviewing this work. Many of the results given in this paper were initially discovered experimentally using the open-source mathematical software Sage [sage] and Sage-Combinat [sage-combinat]. The shin basis was first uncovered by computational experiments of Chris Berg [Berg] and the authors are indebted to him for sharing this discovery and giving them the opportunity to explore its properties. The authors were supported by the Natural Sciences and Engineering Resource Council of Canada; Karen Feldman was supported by an NSERC USRA. We would like to thank an anonymous referee for many useful comments. 


\section{Preliminaries}

To establish some notation, we define a composition of a positive integer $n$ as a tuple $\alpha=\left(\alpha_{1}, \alpha_{2}, \ldots, \alpha_{m}\right)$ such that $\sum_{i=1}^{m} \alpha_{i}=n$ and $\left(\alpha_{1}, \alpha_{2}, \ldots, \alpha_{m}\right) \in \mathbb{N}^{m}$ for some $m \in \mathbb{N}$. The notation $\alpha \vDash n$ is used to indicate that $\alpha$ is a composition of $n$, and the length of $\alpha$, denoted $\ell(\alpha)$, is $m$. We adopt the convention whereby $\alpha_{i}=0$ for all indices $i>\ell(\alpha)$. A partition $\lambda=\left(\lambda_{1}, \lambda_{2}, \ldots, \lambda_{\ell(\lambda)}\right)$ of a positive integer $n$ is a composition satisfying the additional property that $\lambda_{1} \geqslant \lambda_{2} \geqslant \ldots \geqslant \lambda_{\ell(\lambda)}$. To indicate that $\lambda$ is a partition of $n$, we write $\lambda \vdash n$.

$\mathcal{C}$ will be used to denote the set of all integer compositions together with the empty composition (), writing ()$\vDash 0$ by convention, with $\mathcal{C}=\left\{\alpha: \alpha \vDash n\right.$ where $\left.n \in \mathbb{Z}_{\geqslant 0}\right\}$. $\mathcal{C}_{n}$ is the set of all compositions of a fixed $n$, with $\mathcal{C}_{n}=\{\alpha: \alpha \vDash n\}$.

NSym may be defined as the free $\mathbb{Q}$-algebra generated by $\left\{H_{1}, H_{2}, \ldots\right\}$, where $H_{n}$ is defined to be of degree $n \in \mathbb{N}$ and may be regarded as a primitive object. For arbitrary $\alpha \in \mathcal{C}$ write $H_{\alpha}=H_{\alpha_{1}} \ldots H_{\alpha_{\ell(\alpha)}}$, and write $H_{()}=1 . H: \mathcal{C} \rightarrow$ NSym will be referred to as the complete homogeneous function, and the complete homogeneous basis of NSym may be defined to be im $H$.

Observe the following graded algebra structure of NSym, letting NSym $_{r}$ denote the set of all homogeneous polynomials in NSym of degree $r$ together with 0 for $r>0$, with $\mathrm{NSym}_{0}=\mathbb{Q}:$

$$
\mathrm{NSym}=\bigoplus_{r \geqslant 0} \mathrm{NSym}_{r}=\bigoplus_{r \geqslant 0} \operatorname{span}_{\mathbb{Q}}\left(\operatorname{im}\left(\left.H\right|_{\mathfrak{C}_{r}}\right)\right) .
$$

The algebra NSym also has a $\mathbb{Q}$-coalgebra structure whereby the coproduct is given by $\Delta\left(H_{j}\right)=\sum_{i=0}^{j} H_{i} \otimes H_{j-i}$. This coproduct is compatible with the product structure of NSym [NCSF] and hence:

$$
\Delta\left(H_{\alpha}\right)=\Delta\left(H_{\alpha_{1}}\right) \Delta\left(H_{\alpha_{2}}\right) \ldots \Delta\left(H_{\alpha_{\ell(\alpha)}}\right) .
$$

Sym may be analogously defined as the free commutative $\mathbb{Q}$-algebra with one generator in each degree:

$$
\text { Sym }=\mathbb{Q}\left[h_{1}, h_{2}, h_{3}, \ldots\right] .
$$

The coproduct is similarly defined so that $\Delta\left(h_{j}\right)=\sum_{i=0}^{j} h_{i} \otimes h_{j-i}$. This coproduct is compatible with the product structure of Sym and hence:

$$
\Delta\left(h_{\lambda}\right)=\Delta\left(h_{\lambda_{1}}\right) \Delta\left(h_{\lambda_{2}}\right) \ldots \Delta\left(h_{\lambda_{\ell(\lambda)}}\right) .
$$

NSym projects onto Sym with the map

$$
\chi\left(H_{\alpha}\right)=h_{\alpha_{1}} h_{\alpha_{2}} \ldots h_{\alpha_{\ell(\alpha)}} .
$$

This map commutes with the product and coproduct operation since it can be shown on the generators of the algebra that

$$
\chi\left(H_{\alpha} H_{\beta}\right)=\chi\left(H_{\alpha}\right) \chi\left(H_{\beta}\right)
$$


and

$$
(\chi \otimes \chi) \circ \Delta\left(H_{r}\right)=\Delta\left(\chi\left(H_{r}\right)\right) .
$$

QSym may be defined as the $\mathbb{Q}$-algebra spanned by elements $M_{\alpha}$ for $\alpha \in \mathcal{C}$ where the monomial basis $\left\{M_{\alpha}\right\}_{\alpha \in \mathcal{C}}$ of QSym is the dual basis to $\left\{H_{\alpha}\right\}_{\alpha \in \mathcal{C}}$. The symmetric functions are self-dual with respect to the pairing $\langle\cdot, \cdot\rangle: \operatorname{Sym} \times \operatorname{Sym} \rightarrow \mathbb{Q}$ where $\left\langle h_{\lambda}, m_{\mu}\right\rangle=\delta_{\lambda, \mu}$. We note that there is a natural inclusion of Sym into QSym where

$$
m_{\lambda}=\sum_{\operatorname{sort}(\alpha)=\lambda} M_{\alpha}
$$

where the sum is over all compositions $\alpha$ such that the weakly decreasing arrangement of the parts of $\alpha$ is equal to the partition $\lambda$. Alternatively, the inclusion of Sym to QSym is the dual to the projection map $\chi:$ NSym $\rightarrow$ Sym that was introduced in Equation (1). This can be seen if we restate Equation (2) as

$$
m_{\lambda}=\sum_{\alpha}\left(\text { coefficient of } h_{\lambda} \text { in } \chi\left(H_{\alpha}\right)\right) M_{\alpha}
$$

and Equation (1) as

$$
\chi\left(H_{\alpha}\right)=\sum_{\lambda}\left(\text { coefficient of } M_{\alpha} \text { in } m_{\lambda}\right) h_{\lambda}=h_{\text {sort }(\alpha)} .
$$

Letting $\alpha$ be a composition, the descent set of $\alpha$ is $D(\alpha)=\left\{\alpha_{1}, \alpha_{1}+\alpha_{2}, \alpha_{1}+\alpha_{2}+\right.$ $\left.\alpha_{3}, \ldots, \alpha_{1}+\alpha_{2}+\ldots+\alpha_{\ell(\alpha)-1}\right\}$ and this defines a bijection between the compositions of $n$ and the set of subsets of $\{1,2,3, \ldots, n-1\}$. The refinement order is the binary relation $\succeq$ defined on $\mathcal{C}_{n}$ so that $\alpha \succeq \beta$ iff $D(\alpha) \subseteq D(\beta)$. We will use the lexicographic order $\leqslant \ell$ on $\mathcal{C}$ when discussing the triangularity of bases. If $\alpha$ and $\beta$ are elements of $\mathcal{C}$, then $\alpha \geqslant \beta$ if $\alpha_{1}>\beta_{1}$, or $\alpha_{1}=\beta_{1}$ and $\left(\alpha_{2}, \ldots, \alpha_{\ell(\alpha)}\right) \geqslant\left(\beta_{2}, \ldots, \beta_{\ell(\beta)}\right)$.

\section{A New Basis of NSym}

The immaculate basis of NSym was recently introduced in [BBSSZ] as an analogue of the Schur basis of Sym and the dual quasi-Schur basis was introduced in [BLvW, HLMvW11a, HLMvW11b]. Both the immaculate basis and the dual quasi-Schur basis can be defined using a multiplicity-free right Pieri rule and it is using this idea that we consider a third analogue of the Schur basis inside of NSym.

We will define the shin function $\boldsymbol{w}: \mathcal{C} \rightarrow$ NSym using a similar multiplicity-free right Pieri rule, based upon the following analogue of horizontal strips for compositions:

Definition 1. Let $\alpha$ and $\beta$ be compositions. Then $\beta$ differs from $\alpha$ by a shin-horizontal strip of size $r$ iff: for all $i \beta_{i} \geqslant \alpha_{i}$ ("containment axiom"), $|\beta|=|\alpha|+r$ and for all indices $i \in \mathbb{N}$, if $\beta_{i}>\alpha_{i}$, then for all $j>i, \beta_{j} \leqslant \alpha_{i}$ ("overhang axiom"). 
Remark 2. We will henceforward use French notation for diagrams, tableaux, and skewtableaux. Using French notation, the first entry of a composition is at the bottom of the diagram of the composition (see Example 4 and Example 5 below). The overhang axiom says that $\beta / \alpha$ has at most one cell per column and cells in $\beta / \alpha$ should not be below (in a row with a lower index) a row of $\alpha$ which is longer than (overhangs) the horizontal coordinate of the cell.

Remark 3. Observe that the overhang axiom as formulated above holds for all indices $i \in \mathbb{N}$. Recall that we are using the convention whereby for $\alpha \in \mathcal{C}$, for all indices $i>\ell(\alpha)$ we have $\alpha_{i}=0$. See Example 5 below.

Example 4. Let $\alpha=(3,1,4,1,5)$ and $\beta=(3,1,4,1,8,1)$. Then $\beta$ differs from $\alpha$ by a shin-horizontal strip of size 4 .

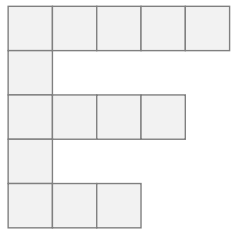

$\operatorname{diag}(\alpha)$

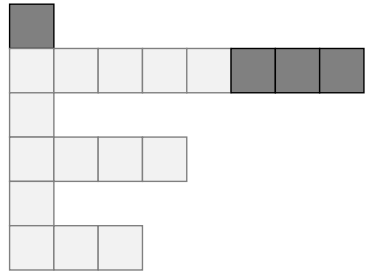

$\operatorname{diag}(\beta)$

Example 5. If $\beta=(3,1,4,1,8,1,1)$, then $\beta$ does not differ from $\alpha=(3,1,4,1,5)$ by a shin-horizontal strip because $\alpha_{6}=0<\beta_{6}=1$ but it is not the case that $\beta_{7} \leqslant \alpha_{6}$ and hence the overhang axiom given in Definition 1 does not hold, as illustrated graphically in the diagram below. If $\gamma=(3,2,4,1,5)$, then $\gamma$ does not differ from $\alpha=(3,1,4,1,5)$ by a shin-horizontal strip because $\gamma_{2}=2>\alpha_{2}=1$ but is is not the case that $\gamma_{3} \leqslant \alpha_{2}$ and hence the overhang axiom does not hold, as illustrated below.
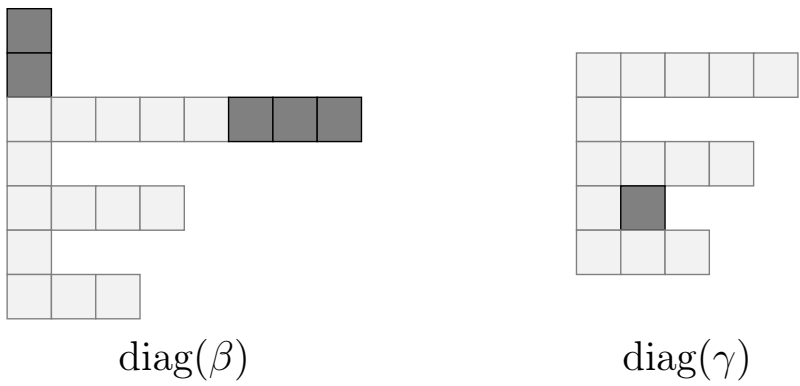

Using Definition 1, we recursively define the shin function as follows:

Definition 6. The shin function $\boldsymbol{E}: \mathcal{C} \rightarrow$ NSym maps an arbitrary composition $\alpha$ to the unique non-commutative symmetric function $\boldsymbol{\varpi}_{\alpha}$ satisfying $\boldsymbol{\varpi}_{\alpha} H_{r}=\sum_{\beta} \boldsymbol{\varpi}_{\beta}$, where the sum is over all compositions $\beta$ which differ from $\alpha$ by a shin-horizontal strip of size $r$.

Remark 7. The interested reader should consult [BBSSZ] and [BLvW] in order to make a proper comparison between the shin basis and the immaculate and dual quasi-Schur bases since they each satisfy a similar right Pieri rule. 
The Pieri rule for the immaculate basis satisfies the containment axiom and the condition that $\ell(\beta) \leqslant \ell(\alpha)+1$.

The Pieri rule for the dual quasi-Schur basis satisfies the containment axiom and the rule that there is at most one cell per column such that if the cells of $\beta / \alpha$ are ordered from left to right then the sequence of indices of the rows is weakly decreasing and cells can only be added on the topmost row of a given length.

A repeated application of the immaculate Pieri rule shows that the complete homogeneous function has a positive uni-triangular expansion in terms of the immaculate function. This property is used to prove that the immaculate basis of NSym is indeed a basis. This leads us to the following analogue of the notion of a column strict tableau, which is very similar to the usual notion of column strict tableaux of partition shape.

Definition 8. Let $\alpha$ and $\beta$ be compositions. A shin-tableau of shape $\alpha$ and content $\beta$ is a labelling of the boxes of the diagram of $\alpha$ by positive integers such that:

1. The number of boxes labeled by $i$ is $\beta_{i}$,

2. The sequence of entries in each row, from left to right, is weakly increasing,

3. The sequence of entries in each column, from lowest index to highest index, is strictly increasing.

Example 9. Observe that when we consider tableaux to be chains of compositions ordered by containment, the overhang axiom is what makes tableaux column-strict. There are 4 shin-tableaux of shape $\alpha=(5,3,1)$ and content $\beta=(3,1,4,1)$ :

\begin{tabular}{|c|c|c|c|}
\hline 4 & & & \\
\hline 3 & 3 & 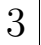 & \\
\hline 1 & 1 & 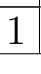 & \begin{tabular}{l|l}
2 & 3 \\
\end{tabular} \\
\hline
\end{tabular}

\begin{tabular}{|c|c|c|c|}
\hline \multicolumn{4}{|l|}{4} \\
\hline 2 & 3 & 3 & \\
\hline 1 & 1 & & \begin{tabular}{l|l}
3 & 3 \\
\end{tabular} \\
\hline
\end{tabular}

\begin{tabular}{|c|c|c|c|}
\hline \multicolumn{4}{|c|}{\begin{tabular}{|l|}
3 \\
\end{tabular}} \\
\hline 22 & 3 & 4 & \\
\hline 1 & 1 & 1 & \begin{tabular}{l|l}
3 & 3 \\
\end{tabular} \\
\hline
\end{tabular}

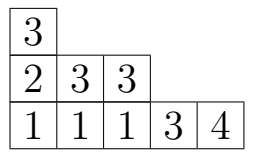

while there are 2 tableaux of shape $(5,1,3)$ and content $(3,1,4,1)$ :

\begin{tabular}{|l|l|l|l|l|}
\hline 3 & \multicolumn{3}{|c|}{3} & 4 \\
\hline 2 & \multicolumn{3}{|c}{} \\
\hline 1 & 1 & 1 & 3 & 3 \\
\hline
\end{tabular}

\begin{tabular}{|c|c|c|c|}
\hline 3 & 3 & 3 & \\
\hline 2 & & & \\
\hline 1 & 1 & & \\
\hline
\end{tabular}

Letting $\mathcal{K}_{\alpha, \beta}$ denote the number of shin-tableaux of shape $\alpha$ and content $\beta$, we arrive at a combinatorial expansion of the complete homogeneous function in the shin basis.

Remark 10. We note that in particular, if $\lambda$ is a partition, then $\mathcal{K}_{\lambda, \beta}$ is equal to the number of column-strict tableaux in the "usual" sense, i.e. $\mathcal{K}_{\lambda, \beta}$ is the Kostka number given by shape $\lambda$ and content $\beta$.

Theorem 11. The complete homogeneous function has a positive, uni-triangular expansion in terms of the shin function. Explicitly,

$$
H_{\beta}=\sum_{\alpha \geqslant \ell \beta} \mathcal{K}_{\alpha, \beta} \ddot{\varpi}_{\alpha}
$$


Proof. This theorem follows from a repeated application of the shin-Pieri rule given in Definition 6 above.

Begin by observing that $H_{\beta_{1}}=\boldsymbol{\varpi}_{\beta_{1}}$, and thus $H_{\beta_{1}} H_{\beta_{2}}=\sum_{\gamma} \boldsymbol{\varpi}_{\gamma}$, where the sum is over all compositions $\gamma$ which differ from $\left(\beta_{1}\right)$ by a shin-horizontal strip of size $\beta_{2}$. Labelling the cells of $\beta_{1}$ with 1's and labelling the added cells with 2's, the sum is over all expressions of the form $\boldsymbol{\Psi}_{\text {shape }(\mathcal{T})}$, where $\mathcal{T}$ is a shin-tableau with content $\left(\beta_{1}, \beta_{2}\right)$. Right-multiply by $H_{\beta_{3}}$ and label the added cells with 3's, then right-multiply by $H_{\beta_{4}}$ and label the added cells with 4's, and so forth.

We can verify by induction against Definition 8 that if $H_{\beta}$ is

$$
H_{\beta}=\sum_{\mathcal{T}} \boldsymbol{\Psi}_{\operatorname{shape}(\mathcal{T})},
$$

where the sum is over shin-tableaux of content $\beta$, then $H_{\beta} H_{r}$ is the sum over shin-tableaux of content $(\beta, r)$.

Note that because the tableaux are strictly increasing in each column and weakly increasing in each row, the first column must be strictly increasing and consequently the cells of label $i$ must be in the first $i$ rows. Hence if $\mathcal{T}$ is of content $\beta$ then $\operatorname{shape}(\mathcal{T}) \geqslant_{\ell} \beta$. Therefore, if $\alpha \leqslant_{\ell} \beta$, then $\mathcal{K}_{\alpha, \beta}=0$.

Also note that $\mathcal{K}_{\alpha, \alpha}=1$, as there is only one filling of the tableau of shape $\alpha$ and content $\alpha$.

It follows from Theorem 11 that, for an arbitrary non-negative integer $n$, the transition matrix from $\left\{\boldsymbol{\Psi}_{\alpha}: \alpha \vDash n\right\}$ to $\left\{H_{\alpha}: \alpha \vDash n\right\}$ is non-singular. We have as a consequence the following corollary.

Corollary 12. $\mathrm{im}(\boldsymbol{\Psi})$ is a basis of NSym.

Definition 13. Let $\alpha$ and $\beta$ be compositions such that $\alpha$ is contained in $\beta$. Let $\beta / \alpha$ denote the cells in $\beta$ that are not in $\alpha$. A filling $\mathcal{T}$ of the cells of $\beta / \alpha$ with natural numbers is a skew shin-tableau if the following two conditions are satisfied:

1. If $\beta_{i}>\alpha_{i}$, then for all $j>i, \alpha_{j} \leqslant \alpha_{i}$ ("overhang axiom");

2. $\mathcal{T}$ is weakly increasing in the rows and strictly increasing in the columns.

We define the skew shape of $\mathcal{T}$ as $\beta / \alpha$, the inner shape of $\mathcal{T}$ as $\alpha$, and the shape of $\mathcal{T}$ as $\beta$.

Example 14. For example, letting $\alpha=(3,1,4,1)$, and letting $\beta=(3,1,4,6,3,1)$, the filling of the cells of $\beta / \alpha$ illustrated below is a skew shin-tableau:

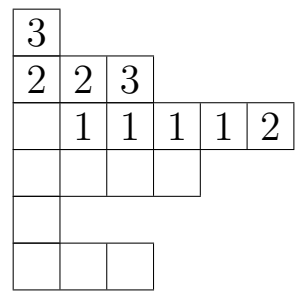


On the other hand, letting $\beta=(3,2,4,1)$, the filling of the cells of $\beta / \alpha$ illustrated below is not a skew shin-tableau, because the overhang axiom does not hold:

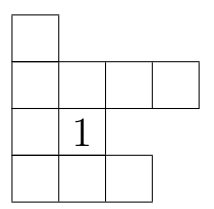

A repeated application of the shin-Pieri rule shows that

$$
\boldsymbol{\Psi}_{\alpha} H_{\beta}=\sum_{\mathcal{T}} \boldsymbol{\Psi}_{\text {shape }(\mathcal{T})},
$$

where the sum is over all skew shin-tableaux with $\beta_{1}$ ones, $\beta_{2}$ twos, etc. Let $\alpha, \beta \in \mathcal{C}$ with $\alpha$ contained in $\beta$. Let $\mathcal{T}$ be a filling of the skew diagram of $\beta / \alpha$ with positive integer labels which are increasing in the columns, letting $\alpha^{(0)}=\alpha$, and letting $\alpha^{(i)}$ denote the underlying composition of the subtableau of $\beta$ as filled by $\mathcal{T}$ with the labels 1 through $i$. Then $\mathcal{T}$ is a skew shin-tableau if the filling of the cells of $\alpha^{(i)} / \alpha^{(i-1)}$ is a shin-horizontal strip for each $i$. For example, the filling of the skew diagram pictured below is a skew shin-tableau:

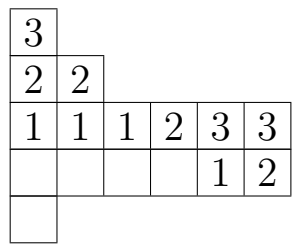

Definition 15. The shin basis of NSym is im(ש).

Let $\chi:$ NSym $\rightarrow$ Sym denote the forgetful mapping whereby for arbitrary $F \in$ NSym, $\chi(F)$ is the commutative image of $F$. The map is defined so that

$$
\chi\left(H_{\alpha}\right)=h_{\alpha_{1}} h_{\alpha_{2}} \ldots h_{\alpha_{\ell(\alpha)}} .
$$

One reason that we focused on this definition of the $\boldsymbol{w}$ basis is that experiments by Chris Berg [Berg] showed that the projection of a shin function was either a Schur function or 0 . In Section 7 we present the dual shin basis of the quasi-symmetric functions and prove the projection formula

$$
\chi\left(\boldsymbol{\Psi}_{\alpha}\right)= \begin{cases}s_{\alpha} & \text { if } \alpha \text { is a partition } \\ 0 & \text { otherwise }\end{cases}
$$

using the duality of non-commutative symmetric functions with the quasi-symmetric functions (see Theorem 28). 


\section{A Formula for Multiplication by a Ribbon}

We begin this section by defining two additional bases of NSym.

Definition 16. The elementary generator indexed by $n$ is recursively defined as $E_{()}=H_{()}$ and for $n \geqslant 1$,

$$
E_{n}=\sum_{i=1}^{n}(-1)^{i+1} H_{i} E_{n-i}=\sum_{\beta \models n}(-1)^{\ell(\beta)+n} H_{\beta} .
$$

For an arbitrary composition $\alpha$, write $E_{\alpha}=E_{\alpha_{1}} E_{\alpha_{2}} \ldots E_{\alpha_{\ell(\alpha)}}$. The elementary basis of NSym is defined to be $\left\{E_{\alpha}: \alpha \in \mathcal{C}\right\}$.

Definition 17. The ribbon function $R: \mathcal{C} \rightarrow$ NSym is defined for a nonempty composition $\alpha$ as follows:

$$
R_{\alpha}=\sum_{\beta \succeq \alpha}(-1)^{\ell(\alpha)-\ell(\beta)} H_{\beta}
$$

It is also the case that $H_{\alpha}=\sum_{\beta \succ \alpha} R_{\beta}$. See [NCSF] for an introduction to the ribbon basis of NSym, and for properties such as $R_{1^{n}}=E_{n}$, and the ribbon multiplication formula:

$$
R_{\alpha} R_{\beta}=R_{\alpha, \beta}+R_{\alpha_{1}, \alpha_{2}, \ldots, \alpha_{\ell(\alpha)}+\beta_{1}, \beta_{2}, \ldots, \beta_{\ell(\beta)}} .
$$

The following theorem uses the concept of skew shin-tableaux as defined in Definition 13. Letting $\mathcal{T}$ be a standard filling of a skew composition of shape $\beta / \alpha$, where $\alpha$ is contained in $\beta$, the descent set of $\mathcal{T}$ is $D(\mathcal{T})=\{i: i+1$ lies above $i\}$.

Theorem 18. For all $\alpha, \beta \in \mathcal{C}$,

$$
\dddot{\varpi}_{\alpha} R_{\beta}=\sum_{\gamma \models|\alpha|+|\beta|} \sum_{\mathcal{T}} \uplus_{\gamma}
$$

where the inner sum is over all standard skew shin-tableaux $\mathcal{T}$ of skew shape $\gamma / \alpha$ such that $D(\mathcal{T})=D(\beta)$.

Proof. Letting $\alpha$ be an arbitrary composition and $m$ be an arbitrary integer, we have $\boldsymbol{\Psi}_{\alpha} R_{m}=\boldsymbol{\varpi}_{\alpha} H_{m}$. By Definition $6, \boldsymbol{\varpi}_{\alpha} H_{m}=\sum_{\gamma} \boldsymbol{\varpi}_{\gamma}$, where $|\gamma|=|\alpha|+m$, and $\gamma$ differs from $\alpha$ by a shin-horizontal strip of size $m$. Filling the horizontal strip with content $\{1,2, \ldots, m\}$ from left to right and from top to bottom, the above conditions hold.

We proceed by induction on the length of $\beta$. Suppose that for some natural number $k$ and for any composition $\beta$ such that $\ell(\beta) \leqslant k, \boldsymbol{\Psi}_{\alpha} R_{\beta}=\sum_{\mathcal{T}} \boldsymbol{\Psi}_{\gamma}$, where the sum is over all standard skew shin-tableaux $\mathcal{T}$ of inner shape $\gamma / \alpha$ such that $D(\mathcal{T})=D(\beta)$, where $\gamma \vDash|\alpha|+|\beta|$. For $\ell(\beta)=k+1$, by the ribbon multiplication rule, we have:

$$
\begin{aligned}
\boldsymbol{\Psi}_{\alpha} R_{\beta} & =\boldsymbol{\varpi}_{\alpha}\left[R_{\left(\beta_{1}, \beta_{2}, \ldots, \beta_{k}\right)} R_{\beta_{k+1}}-R_{\left(\beta_{1}, \beta_{2}, \ldots, \beta_{k}+\beta_{k+1}\right)}\right] \\
& =\boldsymbol{\varpi}_{\alpha} R_{\left(\beta_{1}, \beta_{2}, \ldots, \beta_{k}\right)} H_{\beta_{k+1}}-\boldsymbol{\varpi}_{\alpha} R_{\left(\beta_{1}, \beta_{2}, \ldots, \beta_{k}+\beta_{k+1}\right)} .
\end{aligned}
$$


By our inductive hypothesis, we have:

$$
\boldsymbol{\varpi}_{\alpha} R_{\beta}=\left(\sum_{\mathcal{T}_{1}} \boldsymbol{\Psi}_{\text {shape }\left(\mathcal{T}_{1}\right)}\right) H_{\beta_{k+1}}-\sum_{\mathcal{T}_{2}} \boldsymbol{\Psi}_{\text {shape }\left(\mathcal{T}_{2}\right)} .
$$

where the first sum is over standard fillings $\mathcal{T}_{1}$ with $D\left(\mathcal{T}_{1}\right)=D\left(\beta_{1}, \beta_{2}, \ldots, \beta_{k}\right)$ and the second sum is over standard fillings $\mathcal{T}_{2}$ with $D\left(\mathcal{T}_{2}\right)=D\left(\beta_{1}, \beta_{2}, \ldots, \beta_{k}+\beta_{k+1}\right)$. By Definition 6 , we have:

$$
\left(\sum_{\mathcal{T}_{1}} \boldsymbol{\Psi}_{\text {shape }\left(\mathcal{T}_{1}\right)}\right) H_{\beta_{k+1}}=\sum_{\mathcal{T}} \boldsymbol{\Psi}_{\text {shape }(\mathcal{T})}+\sum_{\mathcal{T}_{2}} \boldsymbol{\Psi}_{\text {shape }\left(\mathcal{T}_{2}\right)}
$$

where the first sum is over all standard fillings $\mathcal{T}$ with $D(\mathcal{T})=D\left(\beta_{1}, \beta_{2}, \ldots, \beta_{k}, \beta_{k+1}\right)$ and the second sum over $\mathcal{T}_{2}$ is exactly the same as that which appears in Equation (3) with $D\left(\mathcal{T}_{2}\right)=D\left(\beta_{1}, \beta_{2}, \ldots, \beta_{k}+\beta_{k+1}\right)$. From Equation (3) and (4) we have for $\ell(\beta)=k+1$, $\boldsymbol{\Psi}_{\alpha} R_{\beta}=\sum_{\mathcal{T}} \boldsymbol{w}_{\text {shape }(\mathcal{T})}$, where the sum is over all standard skew shin-tableaux $\mathcal{T}$ of inner shape $\gamma / \alpha$ such that $D(\mathcal{T})=D(\beta)$, where $\gamma \vDash|\alpha|+|\beta|$.

The above multiplication rule allows us to evaluate shin functions of the form $\boldsymbol{\Psi}_{\left(n, 1^{m}\right)}$ in the ribbon and complete homogeneous basis.

Corollary 19. For an arbitrary composition of the form $\left(n, 1^{m}\right)$,

$$
\boldsymbol{\Psi}_{\left(n, 1^{m}\right)}=R_{\left(n, 1^{m}\right)}=\sum_{\alpha \models n+m, \alpha_{1} \geqslant n}(-1)^{m+1-\ell(\alpha)} H_{\alpha} .
$$

Proof. There is precisely one standard filling of the composition $\left(n, 1^{m}\right)$ with $D(\mathcal{T})=$ $\left(n, 1^{m}\right)$, namely, the one that has $\{1,2, \ldots, n\}$ in the first row and $\{n+1, n+2, \ldots, n+m\}$ in the first column, hence $R_{()} R_{\left(n, 1^{m}\right)}=\boldsymbol{\Psi}_{\left(n, 1^{m}\right)}$.

In the next section we will extend this result further and give expansions for the shin function indexed by compositions of the form $\left(1^{n}, m, 1^{r}\right)$.

We remark that Corollary 19 together with Theorem 18 allow us to evaluate expressions of the form $\boldsymbol{\Psi}_{\alpha} E_{\beta}$ in terms of the complete homogeneous basis (using what may be regarded as "shin-vertical strips" by analogy with Definition 1). We will use the ribbon multiplication rule to prove an analogue of the Murnaghan-Nakayama rule.

\section{A Murnaghan-Nakayama-Like Rule}

Definition 20. For $n \in \mathbb{N}$, let

$$
\Psi_{n}=\sum_{i=0}^{n-1}(-1)^{i} R_{\left(1^{i}, n-i\right)}
$$

and let $\Psi_{\alpha}=\Psi_{\alpha_{1}} \Psi_{\alpha_{2}} \cdots \Psi_{\alpha_{\ell(\alpha)}}$ for a nonempty composition $\alpha$, and write $\Psi_{()}=1$. The set $\left\{\Psi_{\alpha}\right\}_{\alpha \in \mathcal{C}}$ is a basis of NSym [NCSF], referred to as the $\Psi$-basis. 
Remark 21. The $\Psi$-basis is one of the most common analogues of the power sum basis $\left\{p_{\lambda}\right\}_{\lambda \in \mathcal{P}}$ of Sym. Observe that $\chi\left(\Psi_{n}\right)=p_{n}$ for all $n \in \mathbb{N}$.

Definition 22. Let $\alpha$ and $\beta$ be compositions such that $\alpha$ is contained in $\beta$, and if $\beta_{i}>\alpha_{i}$ for arbitrary $i$, then for all $j>i, \beta_{j} \leqslant \alpha_{i}$ (satisfies the overhang axiom). Let $r$ be one less than the number of non-empty rows occupied by $\beta / \alpha$. Label the cells of $\beta /$ $\alpha$ as follows: order the first cell of each row except the last row from bottom to top with $\left[\left(x_{1}, y_{1}\right),\left(x_{2}, y_{2}\right), \ldots,\left(x_{r}, y_{r}\right)\right]$, and order the rest of the cells from left to right with $\left[\left(x_{r+1}, y_{r+1}\right),\left(x_{r+2}, y_{r+2}\right), \ldots,\left(x_{n}, y_{n}\right)\right]$. The skew composition $\beta / \alpha$ is called a shin-slinky of size $n$ and height $r+1$ if and only if for all $i$ satisfying $r+1 \leqslant i<n, y_{i}+1=y_{i+1}$ and $y_{r+1} \leqslant y_{r} \leqslant y_{1} \leqslant y_{n}$.

For a tableau, let $c_{(x, y)}$ denote the label of the cell in the $x^{\text {th }}$ row and $y^{\text {th }}$ column of $\operatorname{diag}(\beta)$. Let $\mathcal{T}(\beta / \alpha)$ be the filling of the skew diagram with the labels $c_{\left(x_{i}, y_{i}\right)}=i$.

Example 23. The skew compositions illustrated below are shin-slinkies, where the Young tableaux to the right indicates the labelling of the skew diagram $\mathcal{T}(\beta / \alpha)$ where $\beta$ is the shape and $\alpha$ is the inner shape:
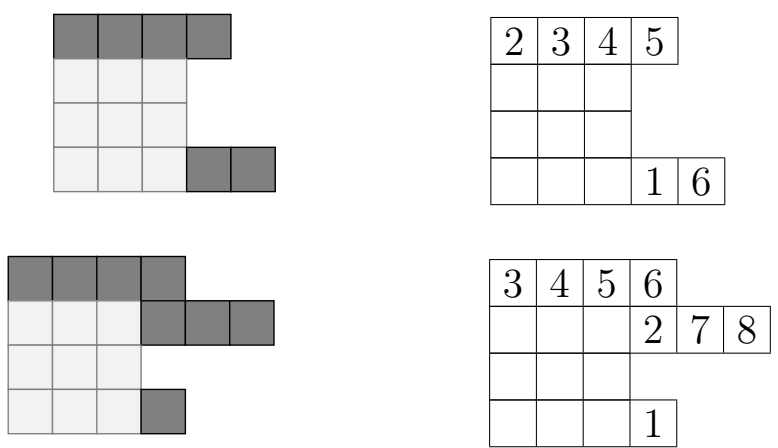

Example 24. The skew compositions illustrated below are not shin-slinkies, where the Young tableau to the right indicates the labelling of the skew composition:
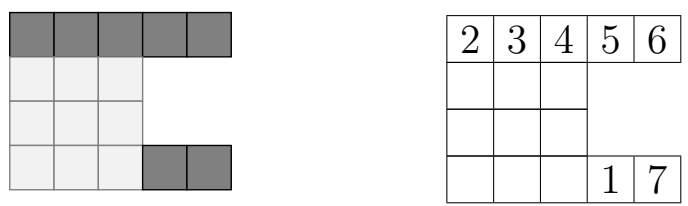

This skew composition is not a shin-slinky, since $y_{6}+1>y_{7}$ for cells $c_{\left(x_{6}, y_{6}\right)}$ and $c_{\left(x_{7}, y_{7}\right)}$.
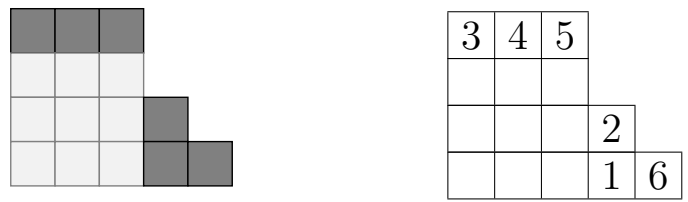

This skew composition is not a shin-slinky, since $y_{5}+1<y_{6}$ for cells $c_{\left(x_{5}, y_{5}\right)}$ and $c_{\left(x_{6}, y_{6}\right)}$. 
Theorem 25. (A Murnaghan-Nakayama rule for the shin basis) Letting $\alpha \in \mathcal{C}$ and $n \in \mathbb{N}$ be arbitrary,

$$
\boldsymbol{\varpi}_{\alpha} \Psi_{n}=\sum_{\beta}(-1)^{\operatorname{height}(\beta / \alpha)-1} \boldsymbol{\varpi}_{\beta},
$$

where the sum is over all compositions $\beta \vDash|\alpha|+n$ such that $\beta / \alpha$ is a shin-slinky. Furthermore, if $\beta / \alpha$ is a shin-slinky, then there is a unique standard filling $\mathcal{T}$ of $\beta / \alpha$ such that $D(\mathcal{T})$ is one of: $\varnothing,\{1\},\{1,2\}, \ldots$, or $\{1,2, \ldots, n-1\}$.

Proof. Substituting $\Psi_{n}=\sum_{i=0}^{n-1}(-1)^{i} R_{\left(1^{i}, n-i\right)}$ into $\boldsymbol{\Psi}_{\alpha} \Psi_{n}$, we have

$$
\boldsymbol{\Psi}_{\alpha} \Psi_{n}=\sum_{i=0}^{n-1}(-1)^{i} \boldsymbol{\Psi}_{\alpha} R_{\left(1^{i}, n-i\right)},
$$

and by Theorem 18, we have

$$
\sum_{i=0}^{n-1}(-1)^{i} \boldsymbol{\Psi}_{\alpha} R_{\left(1^{i}, n-i\right)}=\sum_{i=0}^{n-1}(-1)^{i}\left(\sum_{\mathcal{T}} \boldsymbol{\Psi}_{\operatorname{shape}(\mathcal{T})}\right)
$$

where the inner sum is over all standard fillings $\mathcal{T}$ of inner shape $\alpha$ such that $\operatorname{shape}(\mathcal{T}) \vDash$ $|\alpha|+n$ and $D(\mathcal{T})=\varnothing$ if $i=0$ or $D(\mathcal{T})=\{1,2, \ldots, i\}$ for $i \in[1, n-1]$.

Let $\beta$ be the shape of $\mathcal{T}$ for some standard filling that appears in the right hand side of (6). Order the cells of $\beta / \alpha$ as follows: take the first cell of each row except the last row from bottom to top by $A_{\beta}=\left[\left(x_{1}, y_{1}\right),\left(x_{2}, y_{2}\right), \ldots,\left(x_{r}, y_{r}\right)\right]$ and then order the rest of the cells from left to right and from top to bottom by $B_{\beta}=\left[\left(x_{r+1}, y_{r+1}\right),\left(x_{r+2}, y_{r+2}\right), \ldots,\left(x_{n}, y_{n}\right)\right]$ where $r$ is the number of rows occupied by the skew shape $\beta / \alpha$ minus 1 . Since $D(\mathcal{T})=$ $\{1,2, \ldots, i\}$ for some $i$, each of these labels $\{1,2, \ldots, i+1\}$ must occur in different rows and the rows that they occur in must increase, that is $x_{a_{1}}<x_{a_{2}}<\ldots<x_{a_{i+1}}$ and hence $\left\{\left(x_{a_{1}}, y_{a_{1}}\right),\left(x_{a_{2}}, y_{a_{2}}\right), \ldots,\left(x_{a_{i}}, y_{a_{i}}\right)\right\} \subseteq A_{\beta}$ and $i \leqslant r$. Moreover the labels $\{i+1, i+2, \ldots, n\}$ must not be descents and in particular,

$$
\left\{c_{\left(x_{r+1}, y_{r+1}\right)}<c_{\left(x_{r+2}, y_{r+2}\right)}<\ldots<c_{\left(x_{n}, y_{n}\right)}\right\} \subseteq\{i+1, i+2, \ldots, n\} .
$$

The cells in $B_{\beta}$ are chosen so that $y_{d} \leqslant y_{d+1}$ for $r+2 \leqslant d \leqslant n$. If $y_{d}=y_{d+1}$, then the column strict condition implies $c_{\left(x_{d}, y_{d}\right)}>c_{\left(x_{d+1}, y_{d+1}\right)}$. Therefore, $y_{d}<y_{d+1}$.

Now fix $\beta$ and we will determine the number of fillings of that shape with descent set $\{1,2, \ldots, m\}$. Let $C_{\beta}=\left\{\left(x_{j_{1}} y_{j_{1}}\right),\left(x_{j_{1}} y_{j_{1}}\right), \ldots,\left(x_{j_{s}} y_{j_{s}}\right)\right\}$ be the set of cells in $A_{\beta}$ that do not have a cell in $B_{\beta}$ above. While it is possible that the set $C_{\beta}$ is empty, if there is a $r+1 \leqslant j \leqslant n$ such that $y_{j}+1<y_{j+1}$, then $C_{\beta}$ contains at least one cell in column $y_{j}+1$. Also if $y_{1}>y_{n}$ then $C_{\beta}$ will be non-empty.

Finally, we note that for the descent set $\{1,2, \ldots, m\}$, there are $\left(\begin{array}{c}s \\ m\end{array}\right)$ ways of choosing $m$ of the cells to put the labels $\{1,2, \ldots, m\}$ and the remaining labels $m+1$ through $n$ are uniquely filled into the cells such that they do not have a descent. For $m \in[0, s]$, there are $\left(\begin{array}{c}s \\ m\end{array}\right)$ such fillings for $\beta / \alpha$ with $D(\mathcal{T})=\{1,2, \ldots, m\}$. Since for $s>0, \sum_{m=0}^{s}(-1)^{m+1}\left(\begin{array}{c}s \\ m\end{array}\right)=$ 
0 the coefficient of $\boldsymbol{\Psi}_{\beta}$ will be 0 if $C_{\beta}$ is non-empty. In particular if $y_{j}+1<y_{j+1}$ for some $j$ with $r+1 \leqslant j \leqslant n$.

So the remaining terms all have $C_{\beta}$ empty and $y_{j}+1=y_{j+1}$ for all $r+1 \leqslant j \leqslant n$. We will have that $r=\left|A_{\beta}\right|=$ height $(\beta / \alpha)-1$ and $D(\mathcal{T})=\{1,2, \ldots, r\}$ and the coefficient of $\boldsymbol{\Psi}_{\beta}$ in $\boldsymbol{\Psi}_{\alpha} \Psi_{n}$ is $(-1)^{\text {height }(\beta / \alpha)-1}$.

\section{A Formula for the Shin Function Evaluated at a "Div- ing Board" Composition}

Definition 26. A diving board composition or diving board is a composition of the form $\left(1^{n}, m, 1^{r}\right)$ for $m>1$, as illustrated below:

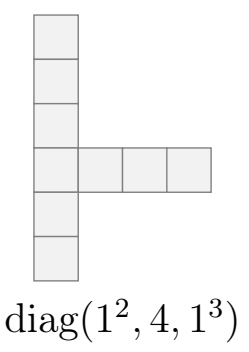

Theorem 27. For $m>1$,

$$
\boldsymbol{\Downarrow}_{\left(1^{n}, m, 1^{r}\right)}=\sum_{i=0}^{r} \sum_{j=0}^{n}(-1)^{i+j} E_{n-j} H_{m+i} H_{j} E_{r-i}
$$

Proof. We begin by examining the base case, where $n=r=0$. In this case the right-hand side of (7) may be simplified as follows, yielding $\boldsymbol{\varpi}_{\left(1^{n}, m, 1^{r}\right)}$ :

$$
\sum_{i=0}^{0} \sum_{j=0}^{0}(-1)^{i+j} E_{0-j} H_{m+i} H_{j} E_{0-i}=(-1)^{0} E_{0} H_{m} H_{0} E_{0}=H_{m}=\boldsymbol{\varpi}_{m} .
$$

Assume that for all compositions of the form $\left(1^{n^{\prime}}, m^{\prime}, 1^{r^{\prime}}\right)$, Equation (7) is true for all $n^{\prime}<n$, or $n^{\prime}=n$ and $r^{\prime}<r$. Then there are two cases we must consider: where $r=0$ and where $r>0$.

For the case where $r=0$,

$$
\boldsymbol{\Psi}_{\left(1^{n}, m\right)}=E_{n} H_{m}-\sum_{k=0}^{n-1} \boldsymbol{\Psi}_{\left(1^{k}, m+1,1^{n-k-1}\right)}-\sum_{k=0}^{n-1} \boldsymbol{\Psi}_{\left(1^{k}, m, 1^{n-k}\right)}
$$

For the case where $r>0$, we will use the Pieri rule for $\boldsymbol{\varpi}_{\alpha} E_{r}$, which says that:

$$
\boldsymbol{\Psi}_{\left(1^{n}, m, 1^{r}\right)}=\boldsymbol{\Psi}_{\left(1^{n}, m\right)} E_{r}-\boldsymbol{\Psi}_{\left(1^{n}, m+1,1^{r-1}\right)}
$$


Before proceeding we will require the following calculation as a step in the proof and so we note that if we start with

$$
\sum_{i=0}^{n-k-1} \sum_{j=0}^{k}(-1)^{i+j} E_{k-j} H_{m+i+1} H_{j} E_{n-k-1-i}+\sum_{i=0}^{n-k} \sum_{j=0}^{k}(-1)^{i+j} E_{k-j} H_{m+i} H_{j} E_{n-k-i},
$$

then we can substitute $i=i^{\prime}-1$. Therefore the sum is equal to:

$$
\sum_{i^{\prime}=1}^{n-k} \sum_{j=0}^{k}(-1)^{i^{\prime}+j-1} E_{k-j} H_{m+i^{\prime}} H_{j} E_{n-k-i^{\prime}}+\sum_{i^{\prime}=0}^{n-k} \sum_{j=0}^{k}(-1)^{i^{\prime}+j} E_{k-j} H_{m+i^{\prime}} H_{j} E_{n-k-i^{\prime}} .
$$

Note that the two double sums have opposite signs; therefore the terms with $i \geqslant 1$ cancel. Thus we are left with the following single sum:

$$
\sum_{j=0}^{k}(-1)^{j} E_{k-j} H_{m} H_{j} E_{n-k}
$$

Case 1: $r=0$. Our inductive hypothesis says that by Equation (7) and Equation (10),

$$
\begin{aligned}
\boldsymbol{\Psi}_{\left(1^{n}, m\right)}= & E_{n} H_{m}-\sum_{k=0}^{n-1}\left(\boldsymbol{\Psi}_{\left(1^{k}, m+1,1^{n-k-1}\right)}+\boldsymbol{\Psi}_{\left(1^{k}, m, 1^{n-k}\right)}\right) \\
= & E_{n} H_{m}-\sum_{k=0}^{n-1}\left(\sum_{i=0}^{n-k-1} \sum_{j=0}^{k}(-1)^{i+j} E_{k-j} H_{m+i+1} H_{j} E_{n-k-1-i}+\right. \\
& \left.\sum_{i=0}^{n-k} \sum_{j=0}^{k}(-1)^{i+j} E_{k-j} H_{m+i} H_{j} E_{n-k-i}\right) \\
= & E_{n} H_{m}-\sum_{k=0}^{n-1}\left(\sum_{j=0}^{k}(-1)^{j} E_{k-j} H_{m} H_{j} E_{n-k}\right)
\end{aligned}
$$

We can extend the above double sum to an infinite sum by subtracting out the $n^{\text {th }}$ term (for $k \geqslant n+1$, all terms are zero).

$$
=E_{n} H_{m}-\sum_{k \geqslant 0} \sum_{j \geqslant 0}(-1)^{j} E_{k-j} H_{m} H_{j} E_{n-k}+\sum_{j \geqslant 0}(-1)^{j} E_{n-j} H_{m} H_{j}
$$

We now substitute $k=k^{\prime}+j$ and switch the order of the sums:

$$
=E_{n} H_{m}-\sum_{j \geqslant 0} \sum_{k^{\prime} \geqslant 0}(-1)^{j} E_{k^{\prime}} H_{m} H_{j} E_{n-k^{\prime}-j}+\sum_{j \geqslant 0}(-1)^{j} E_{n-j} H_{m} H_{j}
$$


We can switch back the order of the sums and rewrite as follows:

$$
=E_{n} H_{m}-\sum_{k^{\prime} \geqslant 0} E_{k^{\prime}} H_{m} \sum_{j \geqslant 0}(-1)^{j} H_{j} E_{n-k^{\prime}-j}+\sum_{j \geqslant 0}(-1)^{j} E_{n-j} H_{m} H_{j}
$$

The sum $\sum_{j \geqslant 0}(-1)^{j} H_{j} E_{n-k^{\prime}-j}=0$ for $k^{\prime} \neq n$; for $k^{\prime}=n, \sum_{j \geqslant 0}(-1)^{j} H_{j} E_{n-k^{\prime}-j}=1$. We then have:

$$
\boldsymbol{\Psi}_{\left(1^{n}, m\right)}=\sum_{j \geqslant 0}(-1)^{j} E_{n-j} H_{m} H_{j}
$$

This equation is simply Equation (7) evaluated for $r=0$.

Case 2: $r>0$.

$$
\begin{aligned}
\boldsymbol{\Psi}_{\left(1^{n}, m, 1^{r}\right)} & =\boldsymbol{\Psi}_{\left(1^{n}, m\right)} E_{r}-\boldsymbol{\Psi}_{\left(1^{n}, m+1,1^{r-1}\right)} \\
& =\left(\sum_{j \geqslant 0}(-1)^{j} E_{n-j} H_{m} H_{j}\right) E_{r}-\sum_{i=0}^{r-1} \sum_{j=0}^{n}(-1)^{i+j} E_{n-j} H_{m+i} H_{j} E_{r-1-i}
\end{aligned}
$$

The first sum is the $i=r$ term of the second sum; therefore we consolidate the two sums to obtain the following:

$$
\boldsymbol{\uplus}_{\left(1^{n}, m, 1^{r}\right)}=\sum_{i=0}^{r} \sum_{j=0}^{n}(-1)^{i+j} E_{n-j} H_{m+i} H_{j} E_{r-i}
$$

which shows that $\boldsymbol{\Psi}_{\left(1^{n}, m, 1^{r}\right)}$ satisfies Equation (7) for $r>0$. Since $\boldsymbol{\Psi}_{\left(1^{n}, m, 1^{r}\right)}$ satisfies Equation (7) for $r \geqslant 0$, therefore Equation (7) is true by induction.

\section{Relation with the Quasi-Symmetric Functions}

With respect to the pairing $\langle\cdot, \cdot\rangle$ on NSym $\times$ QSym defined on the basis elements so that $\left\langle H_{\alpha}, M_{\beta}\right\rangle=1$ if $\alpha=\beta$ and $\left\langle H_{\alpha}, M_{\beta}\right\rangle=0$ otherwise, the elements that are the graded dual to the shin basis are

$$
\boldsymbol{\Psi}_{\alpha}^{*}=\sum_{\beta} \mathcal{K}_{\alpha \beta} M_{\beta}
$$

because, as a consequence,

$$
\left\langle\boldsymbol{\Psi}_{\alpha}, \boldsymbol{\varpi}_{\beta}^{*}\right\rangle=\sum_{\gamma, \tau} \mathcal{K}_{\alpha \gamma}^{-1} \mathcal{K}_{\beta \tau}\left\langle H_{\gamma}, M_{\tau}\right\rangle=\sum_{\gamma} \mathcal{K}_{\alpha \gamma}^{-1} \mathcal{K}_{\beta \gamma}=\left\{\begin{array}{ll}
1 & \text { if } \alpha=\beta \\
0 & \text { otherwise }
\end{array} .\right.
$$

We noted in Remark 10 that for a partition $\lambda$ and a composition $\beta$ of the same size as $\lambda, \mathcal{K}_{\lambda \beta}$ is equal to the number of column-strict tableaux (by the usual definition of 
column-strict tableaux) of shape $\lambda$ and content $\beta$. It is well known that if $\beta$ and $\tau$ are two compositions such that $\operatorname{sort}(\beta)=\operatorname{sort}(\tau)$, then $\mathcal{K}_{\lambda, \beta}=\mathcal{K}_{\lambda, \tau}$. Therefore,

$$
\boldsymbol{\Psi}_{\lambda}^{*}=\sum_{\beta} \mathcal{K}_{\lambda \beta} M_{\beta}=\sum_{\mu} \mathcal{K}_{\lambda \mu} m_{\mu}=s_{\lambda}
$$

Since $\chi$ is the map which is dual to the inclusion map from Sym in QSym, it follows that

$$
\chi\left(\boldsymbol{\Psi}_{\alpha}\right)=\sum_{\lambda}\left(\text { coefficient of } \boldsymbol{\Psi}_{\alpha}^{*} \text { in } s_{\lambda}\right) s_{\lambda}=\left\{\begin{array}{ll}
s_{\lambda} & \text { if } \alpha=\lambda \in \mathcal{P} \\
0 & \text { otherwise }
\end{array} .\right.
$$

This means that the property that the shin basis projects onto either the Schur function or is equal to 0 comes from the fact that the functions that form the graded dual are equal to the Schur function when indexed by a partition. We conclude with a summary of this argument.

Theorem 28. Let $\left\{A_{\alpha}\right\}_{\alpha \in \mathcal{C}}$ be a basis of NSym and let $\left\{A_{\alpha}^{*}\right\}_{\alpha \models n}$ be the set of dual elements to $\left\{A_{\alpha}\right\}_{\alpha \models n}$ such that $\left\{A_{\alpha}^{*}\right\}_{\alpha \in \mathcal{C}}$ is a basis of QSym. If it is the case that for every partition $\lambda$,

$$
s_{\lambda}=A_{\lambda}^{*},
$$

then

$$
\chi\left(A_{\alpha}\right)=\sum_{\lambda}\left(\text { coefficient of } A_{\alpha}^{*} \text { in } s_{\lambda}\right) s_{\lambda}=\left\{\begin{array}{lc}
s_{\lambda} & \text { if } \alpha=\lambda \in \mathcal{P} \\
0 & \text { otherwise }
\end{array} .\right.
$$

In particular, this is true of the shin basis and this implies that

$$
\chi\left(\boldsymbol{\Psi}_{\alpha}\right)=\left\{\begin{array}{ll}
s_{\alpha} & \text { if } \alpha \text { is a partition } \\
0 & \text { otherwise }
\end{array} .\right.
$$

Moreover, this also implies that the product of two elements of the dual shin basis indexed by partitions has a positive expansion in terms of elements of the dual shin basis also indexed by partitions since all of these elements are in the Schur basis and so must satisfy the Littlewood-Richardson rule. That is,

$$
\boldsymbol{\Psi}_{\lambda}^{*} \boldsymbol{\Psi}_{\mu}^{*}=\sum_{\gamma \vdash|\lambda|+|\mu|} c_{\lambda \mu}^{\gamma} \boldsymbol{\Psi}_{\gamma}^{*}
$$

where the coefficients $c_{\lambda \mu}^{\gamma}$ are the usual Littlewood-Richardson coefficients.

One might conjecture that the product of two elements of the dual shin basis has a positive expansion in terms of the dual shin basis. Unfortunately this is not true in general. The first example where this conjecture fails is in the following product (and all other products whose degree is 5 or less have a non-negative expansion):

$$
\boldsymbol{\Psi}_{1}^{*} \boldsymbol{\Psi}_{13}^{*}=-\boldsymbol{\Psi}_{1112}^{*}+\boldsymbol{\Psi}_{1121}^{*}-\boldsymbol{\Psi}_{122}^{*}+\boldsymbol{\Psi}_{131}^{*}+\boldsymbol{\Psi}_{14}^{*}+\boldsymbol{\Psi}_{23}^{*} \text {. }
$$


The main appealing feature of the shin basis is the simple projection rule given above. We see here that by duality, this implies that Sym is the subalgebra of shin functions indexed by partitions.

The shin basis seems worth studying because the notion of shin-tableaux seems like the natural analogue of standard tableaux and hence is likely to appear in other contexts. Fillings of compositions with labels that increase strictly in the columns seem like simple and intuitive combinatorial objects to define. By contrast, the composition tableaux that appear with immaculate or dual quasi-Schur bases are not immediately clearly related to the usual notion of column strict tableaux and are not the same as column strict tableaux when the shape is a partition.

\section{References}

[Berg] C. Berg, private communication, 2013.

[BBSSZ] C. Berg, N. Bergeron, F. Saliola, L. Serrano and M. Zabrocki. A lift of the Schur and Hall-Littlewood bases to non-commutative symmetric functions. Canadian Journal of Mathematics, 66(3):525-565, 2014. doi:10.4153/CJM-2013-013-0.

[BBSSZ2] C. Berg, N. Bergeron, F. Saliola, L. Serrano, M. Zabrocki. The immaculate basis of the non-commutative symmetric functions, extended abstract. FPSAC 2013. arXiv: 1303.4801.

[BBSSZ3] C. Berg, N. Bergeron, F. Saliola, L. Serrano and M. Zabrocki. Indecomposable modules for the dual immaculate basis of quasi-symmetric functions, to appear. Proc. AMS, 2013. arXiv:1304.1224.

[BBSSZ4] C. Berg, N. Bergeron, F. Saliola, L. Serrano and M. Zabrocki. Multiplicative structures of the immaculate basis of non-commutative symmetric functions. 2013. arXiv: 1305.4700 .

[BLvW] C. Bessenrodt, K. Luoto, and S. van Willigenburg. Skew quasisymmetric Schur functions and noncommutative Schur functions. Adv. in Math., 226:4492-4532, 2011. doi:10.1016/j.aim.2010.12.015.

[Gessel] I. Gessel. Multipartite P-partitions and inner products of skew Schur functions, combinatorics and algebra. Contemp. Math., 34:289-317, 1984.

[HLMvW11a] J. Haglund, K. Luoto, S. Mason, and S. van Willigenburg. Quasisymmetric Schur functions. J. Combin. Theory Ser. A, 118(2):463-490, 2011.

[HLMvW11b] J. Haglund, K. Luoto, S. Mason, and S. van Willigenburg. Refinements of the Littlewood-Richardson rule. Trans. Amer. Math. Soc., 363(3):1665-1686, 2011. doi:10.1090/S0002-9947-2010-05244-4.

[HHL] J. Haglund, M. Haiman and N. Loehr. A combinatorial formula for Macdonald polynomials. J. Amer. Math. Soc., 8:735-761, 2005. 
[LS] A. Lascoux and M.-P. Schützenberger. Keys and standard bases. Tableaux and Invariant Theory, IMA Volumes in Mathematics and its Applications, vol. 19:125$144,1990$.

[MR] C. Malvenuto and C. Reutenauer. Duality between quasi-symmetric functions and the Solomon descent algebra. J. Algebra, 177:967-982, 1995.

[NCSF] I. M. Gelfand, D. Krob, A. Lascoux, B. Leclerc, V. S. Retakh, J. Y. Thibon. Noncommutative symmetrical functions. Adv. in Math., 112(2):218-348, 1995. doi:10.1006/aima.1995.1032.

[sage] W. A. Stein et al. Sage Mathematics Software (Version 4.3.3). The Sage Development Team, 2010, http://www. sagemath.org.

[sage-combinat] The Sage-Combinat community. Sage-Combinat: enhancing Sage as a toolbox for computer exploration in algebraic combinatorics. http://combinat. sagemath.org, 2008. 